\title{
Strain relaxation induced 1-dimensional and 0-dimensional structures: Bi on $\mathrm{Ge}(001)$
}

\author{
H.K. Louwsma, H.J.W. Zandvliet *, B.A.G. Kersten, J. Chesneau, A. van Silfhout, \\ B. Poelsema \\ Faculty of Applied Physics and Centre for Materials Research. University of Twente, P.O. Box 217.7500 AE Enschede, \\ The Netherlands
}

Received 13 November 1996; accepted for publication 6 February 1997

\begin{abstract}
The growth of bismuth on Ge(001) has been studied using scanning tunneling microscopy. Deposition of one-monolayer (ML) $\mathrm{Bi}$ at room temperature and subsequent annealing at $500 \mathrm{~K}$ results in the formation of a well-ordered $(2 \times n)$ vacancy line network oriented perpendicular to the dimer rows. Deposition of two-monolayer $\mathrm{Bi}$ under the same conditions results in the formation of square-shaped pits with depths up to $10 \mathrm{ML}$. The edges of these pits run along the two dimer row directions. Careful analysis of these pits reveal that they mainly consist of (111) and (113) facets. We argue that the morphological transition from the vacancy line network to the square shaped pits pattern is driven by a modification of the surface strain tensor. 1997 Elsevier Science B.V.
\end{abstract}

Keywords: Bismuth; Germanium; Low index single crystal surfaces; Scanning tunneling microscopy; Surface stress: Surface structure, morphology, roughness and topography

The growth of various types of overlayer on semiconductor surfaces has been a topic of intense study over the past decades. The growth of the group $\mathrm{V}$ materials such as $\mathrm{As}, \mathrm{Sb}$ and $\mathrm{Bi}$ on $\mathrm{Si}(001)$ and $\mathrm{Ge}(001)$ surfaces is important for the heteroepitaxy because these materials can be used as surfactants as well as dopants. Deposition of submonolayer coverages of these group V materials on $\mathrm{Si}(001)$ or $\mathrm{Ge}(001)$ surfaces gives rise to dimerisation. It is, however, still a matter of debate whether these group $\mathrm{V}$ atoms grow directly on the surface or whether they exchange place with the

* Corresponding author. Fax: + 315348911101 ; e-mail: h.j.w.zandvliet@tun.utwente.nl original top layer of $\mathrm{Si}$ or Ge. In a recent paper by Park et al. [1] the deposition of $\mathrm{Bi}$ on $\mathrm{Si}(001)$ was studied using scanning tunneling microscopy (STM) and low energy electron diffraction (LEED). These authors report the formation of a well-ordered $(2 \times n)$ pattern after the deposition of $\mathrm{Bi}$ and subsequent annealing. STM images reveal that the $(2 \times n)$ pattern consists of ordered rows of missing dimers oriented perpendicular to the dimer rows. Due to a large misfit between the atomic sizes of $\mathrm{Bi}$ and $\mathrm{Si}$, a compressive strain along the dimer row direction is expected to be present. This compressive strain can be released by the formation of a vacancy line network oriented perpendicular to the substrate dimer row direction. It is expected that also the deposition of 
one-monolayer of $\mathrm{Bi}$ on the closely related $\mathrm{Ge}(001)$ surface will give rise to such a vacancy line network. In this paper we show that this is indeed the case. Furthermore, we also consider the two-monolayer case and found that for this coverage the strain relaxation occurs via the formation of square-shaped pits. We suggest that the absence of dimerisation in the second $\mathrm{Bi}$ layer will give rise to a modification of the surface strain tensor such that the surface is under a compressive strain in both orthogonal directions.

The experiments were performed in an UHV system, equipped with a STM. The base pressure during the experiments was kept below $5 \times 10^{-11}$ mbar. The Ge(001) sample had a miscut angle of $0.8^{\circ}$ towards the [110] direction. The surface of the sample was cleaned by $900 \mathrm{eV} \mathrm{Ar}^{+}$ ion bombardment followed by annealing at temperatures of $800-900 \mathrm{~K}$ for $15 \mathrm{~min}$. The Bi source consisted of a thin $\mathrm{Ta}$ foil cell containing $\mathrm{Bi}$. The $\mathrm{Bi}$ was evaporated from the cell by sending a current through the Ta foil. The deposition rate, as determined with the STM, used in these experiments was $0.15 \pm 0.02 \mathrm{ML} / \mathrm{min}$.

In Fig. 1 a $\mathrm{Ge}(001)$ surface after the deposition of $0.15 \mathrm{ML}$ of $\mathrm{Bi}$ is shown. Nearly all $\mathrm{Bi}$ dimers are positioned on top of the substrate dimer rows and have their dimer bond aligned along the dimer row direction of the underlying substrate. Subsequent imaging of the same area (Figs. la and b) shows that many $\mathrm{Bi}$ dimers have been moved to another position. Note also that many Bi dimers are partially imaged. We observed that the scanning process influences the Bi-dimer diffusion process.

Deposition of $1 \mathrm{ML} \mathrm{Bi}$ on a $\mathrm{Ge}(001)$ substrate at room temperature and subsequent annealing at a temperature of $500 \mathrm{~K}$ for 5 min results in a well ordered vacancy line pattern (Fig. 2). The vacancy lines run perpendicular to the substrate dimer row direction. The formation of a similar vacancy line pattern has also been observed on the $\mathrm{Si}(001)$ surface after the deposition of $\mathrm{Bi}[1], \mathrm{Ge}$ [2] and $\mathrm{Ni}$ [3]. But etching of $\mathrm{Si}(001)$ with $\mathrm{Ar}^{+}[4,5]$ or $\mathrm{Br}_{2}, \mathrm{I}_{2}[6-8]$ and $\mathrm{O}_{2}$ [9] also results in the formation of a vacancy line pattern. The ordering of this vacancy line pattern is driven by a strain-induced long-range repulsive interaction along the dimer

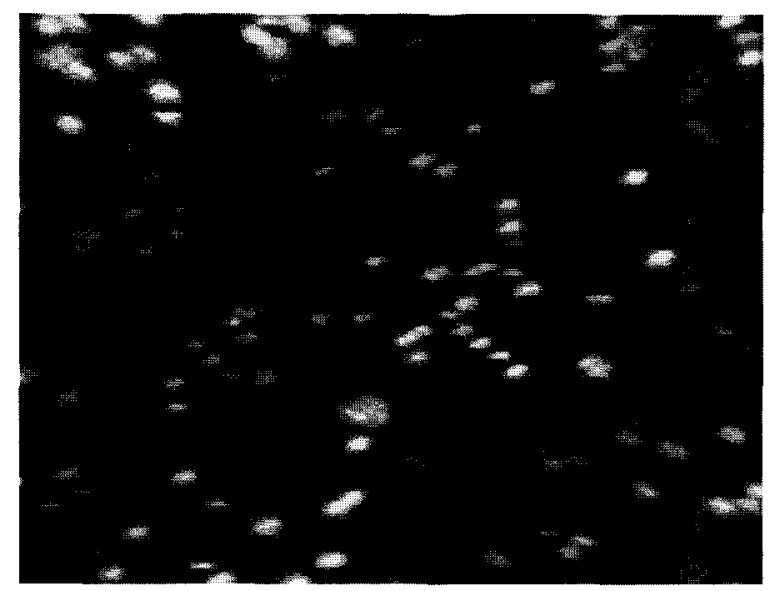

(a)

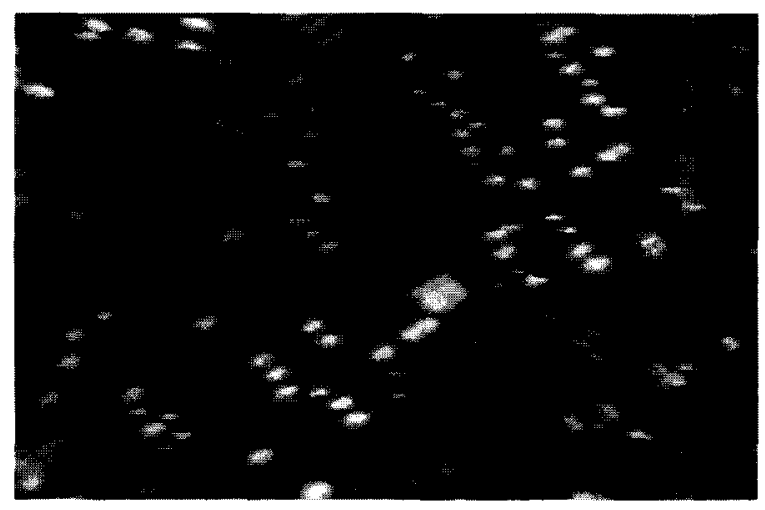

(b)

Fig. 1. Two subsequent images of $\mathrm{Ge}(001)$ covered with about $0.15 \mathrm{ML}$ of $\mathrm{Bi}$ at room temperature. Most of the $\mathrm{Bi}$ dimers are positioned on top of the underlying substrate dimer rows and the $\mathrm{Bi}$ dimers have their dimer bond oriented along the dimer row. The sample bias was $-2 \mathrm{~V}$ and the tunneling current was $1 \mathrm{nA}$. Image size $20 \mathrm{~nm} \times 30 \mathrm{~nm}$.

row direction and a short-range attractive interaction between the vacancies in the direction perpendicular to the dimer row direction. An extensive discussion of this strain-relief induced ordering process can be found in Refs. $[2,10,11]$. Submonolayer coverages of $\mathrm{Bi}$ on $\mathrm{Ge}(001)$ will dimerise and due to the large misfit in atomic radii between $\mathrm{Ge}(1.22 \AA)$ and $\mathrm{Bi}(1.55 \AA)$ a large compressive strain will develop along the substrate dimer row direction [1].

Deposition of about $2 \mathrm{ML} \mathrm{Bi}$ at room temperature, followed by a $5 \mathrm{~min}$ anneal at a temperature of $500 \mathrm{~K}$, results in the surface structure shown in 


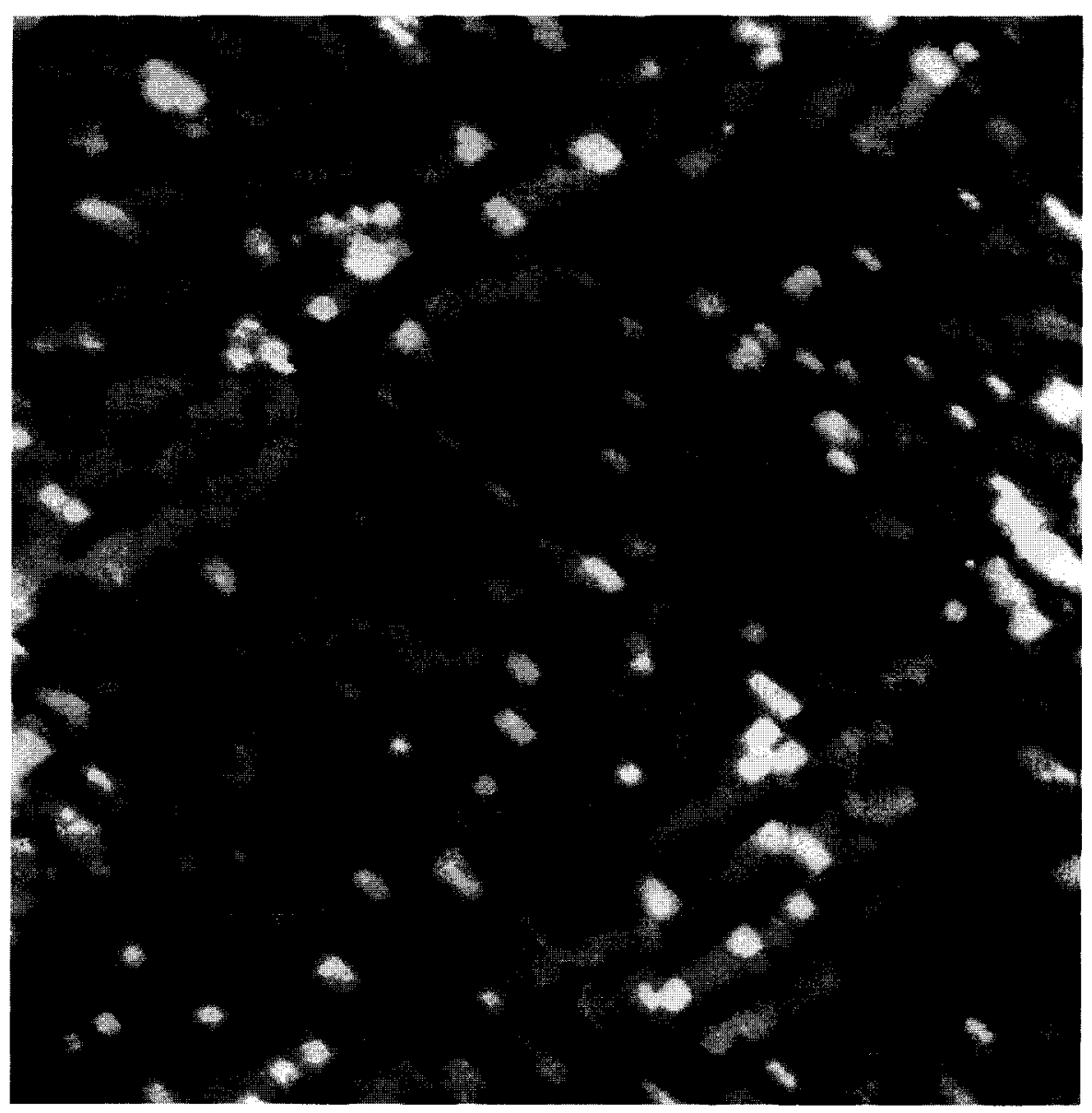

Fig. 2. STM image of a vicinal Ge(001) surface after deposition of $1 \mathrm{ML} \mathrm{Bi}$ at RT and 5 min annealing at $500 \mathrm{~K}$. The sample bias was $-2 \mathrm{~V}$ and the tunneling current was $1 \mathrm{nA}$. Image size $50 \mathrm{~nm} \times 50 \mathrm{~nm}$.

Fig. 3. Several vacancy lines as narrow as one dimer spacing, i.e. $4 \AA$, can still be observed. Most remarkable, however, are the square-shaped pits, which have depths up to about $10 \mathrm{ML}$. The edges of the pits run along the substrate dimer row directions and in most cases a single large pyramid of islands, several monolayers in height, is attached to one of the corners of the pit. A closer inspection of these pits reveals that their shape has the form of a reversed pyramid. The sides of these pyramidshaped defects are mainly composed of the low free-energy $\{111\}$ and $\{113\}$ planes. An increase in the anneal temperature does not change the shape of these square-shaped structures. This simple experiment teaches us that we are dealing with an (at least local) equilibrium structure. If, however, the anneal temperature becomes too high $\mathrm{Bi}$ starts to desorb and a vacancy line pattern (characteristic for the one monolayer regime) develops. A further increase of the anneal temperature to $800 \mathrm{~K}$ finally results in the orginal defect-free surface morphology of clean $\mathrm{Ge}(001)$.

From the depth of the square-shaped pits ( $2 \mathrm{ML}$ regime), which is typically about $5-10 \mathrm{ML}$, we must conclude that these features cannot be the result of the $\mathrm{Bi}$ alone since only $2 \mathrm{ML}$ of $\mathrm{Bi}$ are deposited. Instead, we think that at the location of the pyramidic pits Ge has been removed and is redeposited directly near the pits. This assumption is supported by an inspection of the 3D islands near the edges of the square shaped pits. These island clusters consist of dimerized species at all 


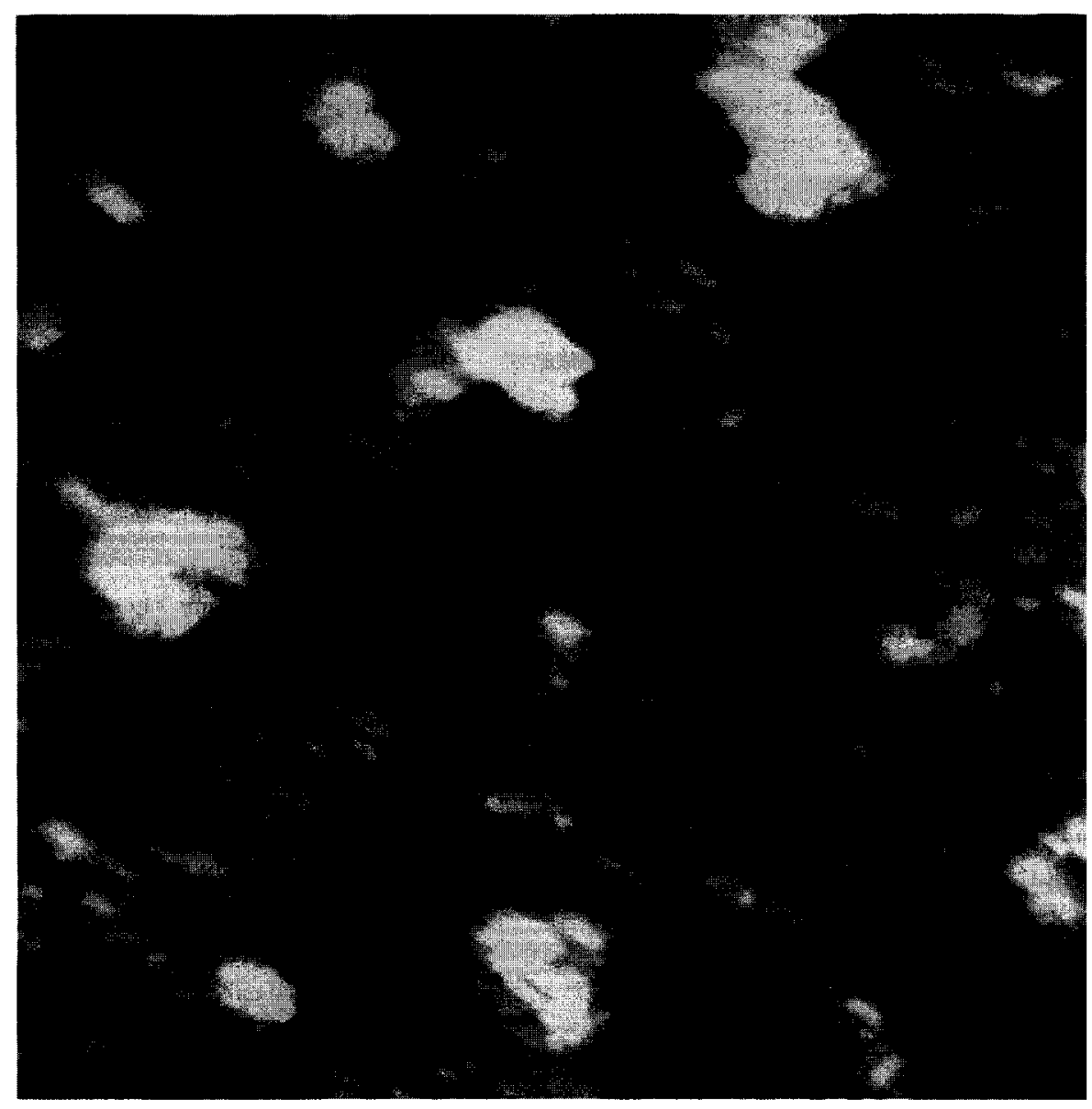

Fig. 3. STM image of a vicinal Ge(001) surface after deposition of $2 \mathrm{ML} \mathrm{Bi}$ at RT and 5 min annealing at $500 \mathrm{~K}$. The sample bias was $-2 \mathrm{~V}$ and the tunneling current was $1 \mathrm{nA}$. Image size $80 \mathrm{~nm} \times 80 \mathrm{~nm}$.

visible levels. Since $\mathrm{Bi}$ is not expected to show dimerisation in islands of these heights, it is reasonable to assume that the $3 \mathrm{D}$ islands consist (at least partly) of Ge. Furthermore, line scans across the pyramids reveal that step heights of about $1.5 \AA$ (which is close to the monatomic step height of $\mathrm{Ge}$, i.e $1.4 \AA$ ) do occur frequently. The appearance of $\mathrm{Ge}$ through pores in the $\mathrm{Bi}$ layer resembles the segregation process which has been observed in the $\mathrm{Co} / \mathrm{Cu}$ system. Recently, Schmid et al. [13] and Giesen et al. [12] observed the formation of similar square-shaped pits after the growth of several monolayers of $\mathrm{Co}$ on flat and vicinal $\mathrm{Cu}(001)$. According to Schmid et al. [13] these pits are formed due to an imperfect layerby-layer growth during the deposition of the first monolayers. During annealing of the films the $\mathrm{Cu}$ diffuses out of the pits on top of the Co surface. The driving force for the diffusion of $\mathrm{Cu}$ onto the Co surface is related to the lower surface free energy of $\mathrm{Cu}$ versus $\mathrm{Co}$. The formation of the square-shaped pits including the island clusters attached to the pit requires substantially more free energy as compared to the flat surface. However, as has been pointed out by Giesen et al. [12], the strain relaxation in the thin Co film due to the formation of these pits is large enough to compensate for the additional energy required to form these pits. This seems a plausible explanation for the $\mathrm{Bi}$ on $\mathrm{Ge}(001)$ system. We should bear in mind, however, that the $\mathrm{Bi} / \mathrm{Ge}(001)$ system is more complicated because the clean as well as the 
Bi-covered $\mathrm{Ge}(001)$ surface will dimerise. The first deposited monolayer of $\mathrm{Bi}$ on $\mathrm{Ge}(001)$ will dimerise, and along with this dimerisation goes a strong compressive strain in the dimer row direction. Because the distance between adjacent dimer rows is twice the distance between adjacent dimers within one row it is reasonable to assume that the surface strain component perpendicular to the dimer rows is significantly smaller. The compressive strain component along the dimer row direction can be released by the formation of a onedimensional vacancy line network as has been discussed previously. After the deposition of $2 \mathrm{ML}$ of $\mathrm{Bi}$ only the top layer can dimerise. The second layer is expected, due to the larger atomic radius of $\mathrm{Bi}$ as compared to $\mathrm{Ge}$, to be under compressive strain in both directions [14]. The most natural way to release this compressive strain is by the formation of holes in the $\mathrm{Bi}$ layer. The compressive strain in the dimerised top layer can still be released by the formation of a vacancy line network. This is precisely what we observe in our STM images. Besides the formation of holes in the Bi layer we also found some vacancy lines. Interestingly, we have found vacancy lines which are aligned perpendicular to, as well as along, the dimer row direction, indicating that the second layer is indeed a Bi layer and not a Ge layer. A more quantitative analysis, such as in the $\mathrm{Co} / \mathrm{Cu}$ case, is not appropriate here because values for the key energetic parameters, such as the elastic constant of $\mathrm{Bi}$ and the surface free energy of $\mathrm{Bi}$-covered $\mathrm{Ge}(001)$ are, at least to our best knowledge, not available from the literature.

In summary, the formation of a vacancy line network after the deposition of $1 \mathrm{ML} \mathrm{Bi}$ on
$\mathrm{Ge}(001)$ as well as the formation of the squareshaped pits after the deposition of $2 \mathrm{ML}$ can, at least qualitatively, be understood in terms of strain relaxation. In the $1 \mathrm{ML}$ case only a compressive strain component along the dimer row direction must be released, whereas in the $2 \mathrm{ML}$ case there is strain relaxation in both orthogonal directions.

\section{References}

[1] Ch. Park, R.Z. Bakhtizin. T. Hashizume, T. Sakurai, J. Vac. Sci. Technol., B 12 (1994) 2049, Jpn. J. Appl. Phys. 32 ( 1993$) 528$.

[2] F. Wu, X. Chen, Z. Zhang, M.G. Lagally, Phys. Rev. Lett. 74 (1995) 574

[3] H. Niehus, U.K. Köhler, M. Copel, J.E. Demuth, J. Micros. 152 (1988) 735.

[4] H.J.W. Zandvliet, H.B. Elswijk, E.J. van Loenen, I.S.T. Tsong, Phys. Rev. B 46 (1992) 7581.

[5] H. Feil, H.J.W. Zandvliet, M.-H. Tsai, J.D. Dow. I.S.T. Tsong. Phys. Rev. Lett. 69 (1992) 3076.

[6] D. Rioux, M. Chander, Y.Z. Li, J.H. Weaver, Phys. Rev. B 49 (1994) 11071.

[7] D. Rioux, R.J. Pechman, M. Chander, J.H. Weaver, Phys. Rev. B 50 (1994) 4430.

[8] D. Rioux, F. Stepniak, R.J. Pechman, J.H. Weaver, Phys. Rev. B 51 (1995) 10981.

[9] K. Wurm, R. Kliese, Y. Hong, B. Röttger, Y. Wei, H. Neddermeyer, I.S.T. Tsong, Phys. Rev. B 50 (1994) 1567.

[10] H.J.W. Zandvliet, H.K. Louwsma, P.E. Hegeman, B. Poelsema, Phys. Rev. Lett. 75 (1995) 3890.

[11] P. Zeppenfeld, M. Krzyzowski, C. Romainczyk, G. Consa, M.G. Lagally, Phys. Rev. Lett. 72 (1994) 2737.

[12] M. Giesen, F. Schmitz, H. Ibach, Surf. Sci. 336 (1995) 269.

[13] A.K. Schmid, D. Atlan, H. Itoh, B. Heinrich, T. Ichinokawa, J. Kirschner, Phys. Rev. B 48 (1993) 2855.

[14] Bi has a rhombohedral structure with a lattice constant of about $4.55 \AA$. 